\title{
CD34+ FIBROBLAST-LIKE CELLS IN THE INTERSTITIAL INFILTRATES IN GLOMERULONEPHRITIS - AN IMMUNOHISTOCHEMICAL OBSERVATION
}

\author{
Cristina Gluhovschi ${ }^{1}$, Elena Potencz ${ }^{2}$, Elena Lazar ${ }^{2}$, Ligia Petrica ${ }^{1}$, Gheorghe Bozdog $^{1}$, \\ Florica Gadalean $^{1}$, Flaviu Bob ${ }^{1}$, Adrian Gluhovschi ${ }^{3}$, Daniel Cioca ${ }^{4}$, Silvia Velciov ${ }^{1}$
}

\author{
${ }^{1}$ Division of Nephrology, University of Medicine and Pharmacy "V. Babes" Timisoara, Romania \\ 2Department of Pathology, University of Medicine and Pharmacy "V. Babes" Timisoara, Romania \\ 3University of Medicine and Pharmacy "V. Babes" Timisoara, Romania, Bega Hospital \\ ${ }^{4}$ Department of Cellular and Molecular Biology, University of Medicine and Pharmacy "V. Babes" Timisoara, Romania
}

\begin{abstract}
CD34 cells in the interstitial infiltrates in glomerulonephritis (GN) could be the turning point between regenerative processes and interstitial fibrosis. The aim of our study was to assess the presence of CD34 + cells in the interstitial infiltrates in GN.

A cross-sectional study of 33 patients with glomerulonephritis, mean age: 43.3 \pm 11.31 years, 20 male and 13 female, was conducted. Conventional stains, as well as immunohistochemistry for the CD34 antigen were employed on kidney biopsies. Strength of immunohistochemical reaction was assessed semi-quantitatively.

Regarding the percentage of cases with CD34+ cells in the interstitial infiltrates out of 33 patients: cells of interstitial infiltrates were $27.3 \%$ positive. The percentage of cases showing CD34+ cells at the level of interstitial infiltrates was: $44.4 \%$ in FSGS, $14.3 \%$ in membranoproliferative GN, $28.6 \%$ in membranous nephropathy, $20 \%$ in mesangial proliferative $\mathrm{GN}, 0 \%$ in minimal change disease, and $50 \%$ in crescentic GN. With the exception of minimal change disease, CD34+ cells were found in the interstitial infiltrates in all histopathological forms of GN. Some of these cells were spindle-shaped fibroblast-like cells. As inflammation in the tubulointerstitial compartment either resolves or proceeds to fibrosis, aims at reversing this process will benefit from analyses of the interstitial infiltrates harboring CD34+ cells.
\end{abstract}

Key words: CD34, glomerulonephritis, fibroblast.

\section{Introduction}

The evidence that fibroblasts regulate the switch from acute resolving to chronic persistent inflammation raises the question of the contribution of CD34+ cells to the fibroblast pool in inflammatory microenvironments. CD34 is a stage-specific rather than lineage-specific marker and has been found on numerous hematopoietic stem cell-progenitor genealogies, endothelial cells and subsets of mesenchymal stells. CD34 cells in the interstitial infiltrates in glomerulonephritis (GN) could be the turning point between regenerative processes and interstitial fibrosis.

It has been known since the seventies that a prominent inflammatory infiltrate within the interstitial compartment precedes fibrosis and progressive decline in renal function. The severity of renal fibrosis is the single best histologic correlate of the decline in renal function and long-term prognosis $[1,2]$.

Fibroblasts play a dominant role in the dynamics of the inflammatory infiltrate by allowing lymphocyte survival and retention. They could represent the turning 
point regulating the switch from acute resolving to chronic persistent inflammation. Chronic inflammation occurs because of disordered fibroblast behavior in which failure to switch off their inflammatory programme leads to the inappropriate survival and retention of leukocytes within inflamed tissue [3, 4]. The inappropriate production of chemokines and matrix components by fibroblasts has dramatic effects on cells of the acquired immune system and may lead to the establishment of chronic inflammation [3].

The CD34 antigen is a marker of hematopoietic stem cells and endothelial cells, but has also been found on fibroblasts. As stated before, CD34+ cells in the interstitial infiltrates in glomerulonephritis could be the turning point between regenerative processes and interstitial fibrosis.

Current anti-inflammatory therapies target immune cells in an attempt to inhibit the production of inflammatory mediators. However, as fibroblasts help orchestrate the inflammatory infiltrate, they could be a valuable target for future anti-inflammatory therapies [5]. Before designing therapies aimed at manipulating fibroblasts, they need to be better characterized. One step in this direction could be defining the contribution of CD34 + cells to the fibroblast pool in inflammatory microenvironments, such as the tubulointerstitium in proteinuric glomerular diseases.

\section{Objectives}

The aim of our study was to assess the presence of CD34+ cells in the interstitial infiltrates in GN.

\section{Material and methods}

A cross-sectional study of 33 patients with glomerulonephritis, mean age: $43.3 \pm 11.31$ years, 20 male and 13 female, was conducted.

All patients underwent percutaneous kidney biopsies after providing informed consent. Conventional stains, as well as immunohistochemistry for the CD34 Class II antigen were employed on kidney biopsies. All biopsy specimens were assessed by two pathologists.

Table I. Number and percentage of cases with CD34+ cells of interstitial infiltrates

\begin{tabular}{ll}
\hline STRENGTH OF & NUMBER AND PERCENTAGE \\
IMMUNOHISTOCHEMICAL & $\begin{array}{l}\text { OF CASES WITH CD34+ } \\
\text { CELLS OF INTERSTITIAL } \\
\text { REACTION }\end{array}$ \\
\hline INFILTRATES \\
\hline $0-$ absent & $24(72.7 \%)$ \\
\hline $1-$ mild & $6(18.2 \%)$ \\
\hline $3-$ moderate & $3(9.1 \%)$ \\
\hline intense & $0(0 \%)$ \\
\hline
\end{tabular}

Sections containing more than eight glomeruli were considered optimal for assessment.

\section{Immunohistochemistry}

The detection of the CD34 Class II antigen was performed on $6 \mu \mathrm{m}$-thick, routinely formalin-fixed paraffin-embedded sections using the horseradish-peroxidaselabeled streptavidin-biotin (EnVision-HRP) method. The primary antibody used was monoclonal mouse anti-human CD34 Class II antigen (Clone QBEnd 10, DAKO). The EnVision-HRP technique was employed as specified by the manufacturer's protocol. Briefly, sections were deparaffinized, rehydrated with distilled water, and pretreated with DakoCytomation Target Retrieval Solution. The next step was serum blocking, followed by incubation with the prediluted primary antibody. Following peroxidase blocking, sections were incubated sequentially with a biotinylated secondary antibody and peroxidase-labeled streptavidin. Labeling was completed by incubating the sections with the chromogenic substrate solution 3-3' diaminobenzidine (DAB). After this, sections were counterstained with Mayer's hematoxylin, rinsed in running tap water, and dehydrated. Sections were then cleared in two changes of toluene and mounted using Eukit. The negative control was run simultaneously with patient specimen.

Normal-appearing tissue from four patients who underwent nephrectomy for suspected kidney tumors served as a control.

Cells of interstitial infiltrates were assessed for immunohistochemical CD34 Class II antigen expression.

The strength of the immunohistochemical reaction was assessed using a semi-quantitative score: $0-a b-$ sent, 1 - mild, 2 - moderate, 3 -intense.

The percentage (frequency count) of patients exhibiting positive CD34 Class II antigen expression was computed.

\section{Results}

The 33 patients presented the following histopathological forms of GN (diagnosis based on light microscopy):

- 9 patients had Focal and Segmental Glomerulosclerosis (FSGS),

- 7 patients: membranoproliferative GN,

- 7 patients: membranous nephropathy,

- 5 patients: mesangial proliferative GN,

- 3 patients: minimal change disease (MCD), and

- 2 patients: crescentic GN.

The number and the percentage of cases with CD34+ cells of interstitial infiltrates out of 33 cases are presented in Table I.

Cells of the interstitial infiltrates were positive in $27.3 \%$ of the cases, with $18.2 \%$ presenting a 1 -mild and $9.1 \%$ presenting a 2-moderate immunohistochemical reaction. 


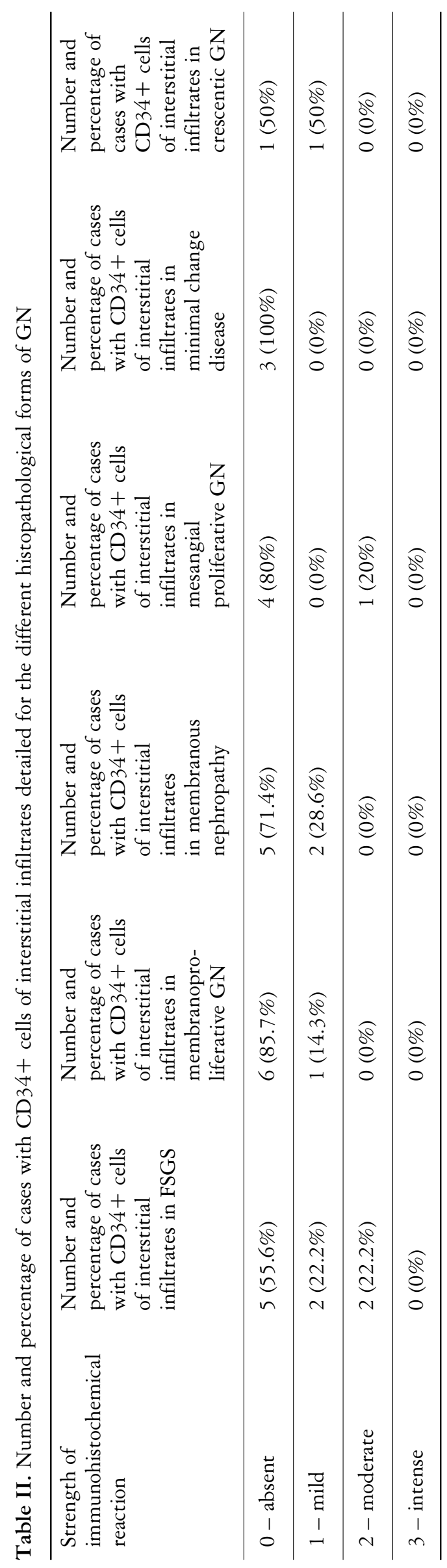

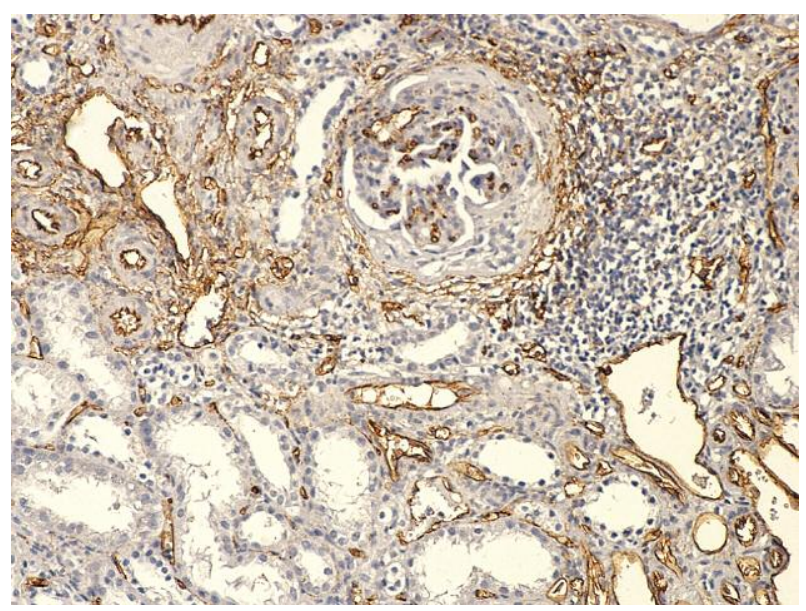

Fig. 1. Partially sclerosed glomerulus, many newlyformed periglomerular capillaries and rich chronic interstitial cell infiltrate. CD34 positive expression in intra- and extraglomerular endothelial cells, in macrophages and fibroblasts. CD34 Stain DAB 200×

The percentage of cases showing CD34+ cells at the level of interstitial infiltrates was: $44.4 \%$ in FSGS (22.2\% presenting a 1 -mild and $22.2 \%$ a 2 -moderate immunohistochemical reaction), $14.3 \%$ in membranoproliferative GN (all presenting a 1-mild immunohistochemical reaction), $28.6 \%$ in membranous nephropathy (all presenting a 1-mild immunohistochemical reaction), $20 \%$ in mesangial proliferative GN (all presenting a 2-moderate immunohistochemical reaction), $0 \%$ in minimal change disease, and $50 \%$ in crescentic GN (all presenting a 1-mild immunohistochemical reaction).

The number and the percentage of cases with CD34+cells of interstitial infiltrates for the different histopathological forms of GN are presented in Table II.

Examples of the immunohistochemical localization of CD34 in cells of the interstitial infiltrates are depicted in Fig. 1 and 2.

It should be emphasized that the identification of cell types was done based on overall morphology.

None of the cases from the control group presented CD34+ cells in the interstitial infiltrates.

\section{Discussion}

One of the unanswered questions in nephrology is: Why do chronic kidney diseases tend to progress? Attempts to answer this question have started with determining the factors related to chronic kidney disease progression. It is known that interstitial fibrosis is the strongest morphologic predictor of clinical outcome and is most tightly linked to progression of disease, even though the primary disease is of glomerular origin. It is also known that fibrosis in the tubulointerstitial com- 


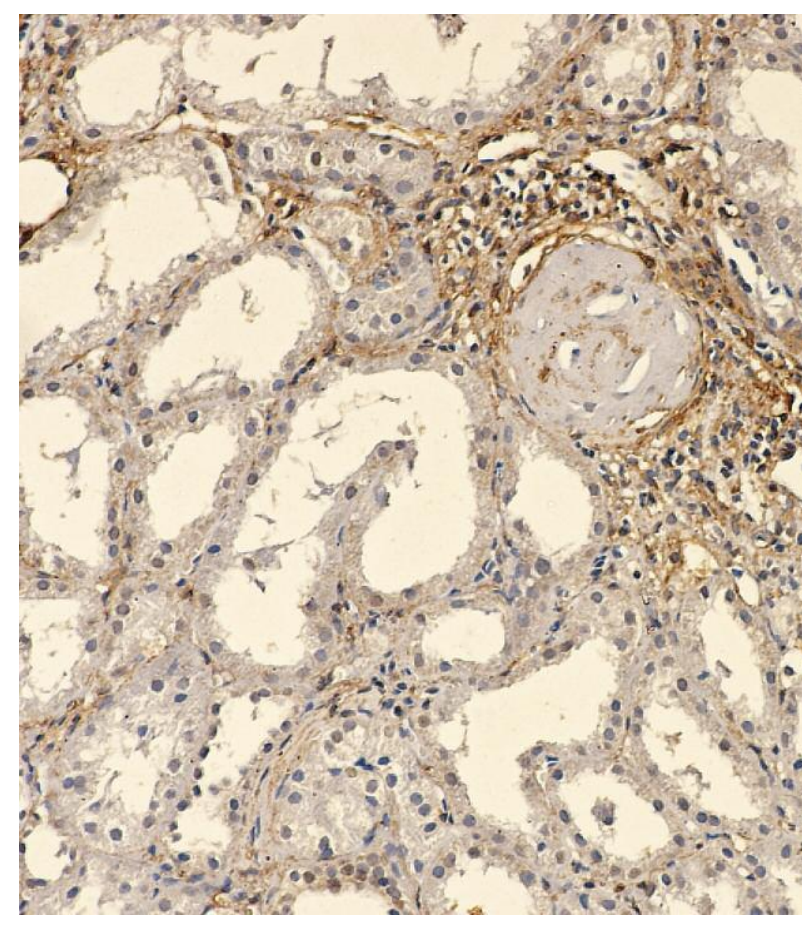

Fig. 2. Hyalinized glomerulus with CD34-negative expression, periglomerular infiltrate with CD34-positive cells, endothelium with CD34 positive expression. CD34 Stain DAB 200X

partment is preceded by inflammation and that the trigger for inflammation is proteinuria.

Inflammatory responses within tissue microenvironments occur with contributions from both hematopoietic and stromal cells. Fibroblasts are not just passive structural landscaping cells, but important sentinel cells of the immune system that help define the inflammatory microenvironment by regulating the switch from acute inflammation to adaptive immunity and tissue repair [3].

CD34 is a cell surface glycoprotein and functions as a cell-cell adhesion factor. It is involved in cell morphogenesis and migration and depending on the circumstances has a dual role in cell-cell adhesion: it can both promote and inhibit cell-cell adhesion by acting as a 'molecular Teflon' [6]. CD34 is found on numerous hematopoietic stem cell-progenitor genealogies, endothelial cells and subsets of mesenchymal stells. CD34 cells in the interstitial infiltrates in glomerulonephritis could be either stem cells participating in regenerative processes or fibroblasts, and as such CD $34+$ cells in the interstitial infiltrates in glomerulonephritis could represent the turning point between regenerative processes and interstitial fibrosis.

In nephropathology, CD34 has been studied as a marker of mesangial activation by Naruse and Chebotareva $[7,8]$. In one of our previous studies we have analyzed the presence of CD34 at the level of the extraglomerular mesangium, concluding that it could be either a marker of stem cells entering the glomerulus at this level or a marker of mesangial activation [9].

In the present study we sought to assess the presence of CD34+ cells in the interstitial infiltrates in GN.

We found that the percentage of cases showing CD34 + cells at the level of interstitial infiltrates was: $44.4 \%$ in FSGS, $14.3 \%$ in membranoproliferative GN, $28.6 \%$ in membranous nephropathy, $20 \%$ in mesangial proliferative $\mathrm{GN}, 0 \%$ in minimal change disease, and $50 \%$ in crescentic GN. Some of the CD34+ cells had a spindle-like shape, as those depicted in Fig. 1 and 2. Whether CD34+ cells in the interstitial infiltrates represent stem cells or fibroblasts, we conclude that their number is not high.

The immunohistochemical observation of spindleshaped CD34 + cells at the level of the interstitial infiltrates in glomerulonephritis needs to be signaled. Further investigations are needed to establish the relative contribution of CD34+ cells to the fibroblast pool in inflammatory microenvironments.

CD34 positive spindle cells have been reported to be present in the interstitium in patients with glomerulonephritis by Okon, El-Koraie and El Kossi [10-12].

By studying 30 renal biopsies of patients with $\mathrm{GN}$, Okon identified CD34+ spindle cells in the renal interstitium in all but one case [10]. In Okon's study CD $34+$ cells were more numerous in membranoproliferative and membranous glomerulopathy, and less numerous in focal and segmental glomerulosclerosis [10].

El-Koraie detected CD34 positive cells as isolated cells in the renal interstitium in patients with GN [11].

El Kossi identified CD34+ cells in the renal interstitium of patients with crescentic GN [12].

Okon states that the pathogenetic significance of CD34 positive spindle cells remains unclear and that at present their possible role can only be speculated [10].

\section{Conclusions}

As fibroblasts are regulating the switch from acute resolving to chronic persistent inflammation, they could represent the turning point between regenerative processes and fibrosis.

As CD $34+$ fibroblast cells could represent a therapeutic checkpoint, further investigations to establish the relative contribution of $\mathrm{CD} 34+$ cells to the fibroblast pool in inflammatory microenvironments, including the interstitial infiltrates in glomerulonephritis, are warranted.

The summary of this paper was presented as a poster at the $48^{\text {th }}$ Congress of the European Renal Association-European Renal Dialysis and Transplant Association, Prague 2011.

Authors declare no conflict of interest. 


\section{References}

1. Risdon RA, Sloper JC, De Wardener HE. Relationship between renal function and histological changes found in renal-biopsy specimens from patients with persistent glomerular nephritis. Lancet 1968; 2: 363-366.

2. Schainuck LI, Striker GE, Cutler RE, Benditt EP. Structuralfunctional correlations in renal disease. II. The correlations. Hum Pathol 1970; 1: 631-641.

3. Buckley CD, Pilling D, Lord JM, et al. Fibroblasts regulate the switch from acute resolving to chronic persistent inflammation. Trends Immunol 2001; 22: 199-204.

4. Buckley CD. Why does chronic inflammation persist: An unexpected role for fibroblasts. Immunol Lett 2011; 138: 12-14.

5. Flavell SJ, Hou TZ, Lax S, et al. Fibroblasts as novel therapeutic targets in chronic inflammation. Br J Pharmacol 2008; 153 (Suppl 1): S241-S246.

6. Nielsen JS, McNagny KM. Novel functions of the CD34 family. J Cell Sci 2008; 121: 3683-3692.

7. Naruse K, Fujieda M, Miyazaki E, et al. CD34 expression as a novel marker of transformed mesangial cells in biopsied glomerular diseases. J Pathol 1999; 189: 105-111.

8. Chebotareva NV, Proppe D, Rudolf P, Kozlovskaia LV. Clinical significance of expression of smooth muscle actin-alpha and CD34 antigen in mesangial cells in glomerulonephritis. Ter Arkh 2002; 74: 27-31.

9. Gluhovschi C, Gluhovschi G, Potencz E, et al. What is the significance of CD34 immunostaining in the extraglomerular and intraglomerular mesangium? Virchows Arch 2008; 453: 321328.

10. Okon K, Szumera A, Kuzniewski M. Are CD34+ cells found in renal interstitial fibrosis? Am J Nephrol 2003; 23: 409-414.

11. El-Koraie AF, Baddour NM, Adam AG. The reparative role of CD34+ cells in human nephropathies. Bull Alex Fac Med 2006; 42: 981-988.

12. El Kossi MM, El Nahas AM. Stem cell factor and crescentic glomerulonephritis. Am J Kidney Dis 2003; 41: 785-795.

\section{Address for correspondence}

\section{Cristina Gluhovschi}

Calea Aradului No. 8 Ap.16

300088 Timisoara

Romania

tel. +40-256-435950

e-mail: gluhovschi@yahoo.com,gluh@umft.ro 